\title{
Analysis of low frequency harmonics for continuous-conduction-mode boost power-factor correction
}

\author{
C.-S.Lin, T.-M.Chen and C.-L.Chen
}

\begin{abstract}
The input current harmonics of continuous-conduction-mode boost power-factor correction (CCMPFC) are presented. Input current distortion of single-phase CCMPFC is analysed and harmonic components are mathematically derived. Upper limits of the boost inductance for CCMPFC are also discussed. Design examples of CCMPFC are demonstrated. Experimental recordings verify the analytical derivations.
\end{abstract}

\section{Introduction}

Power-factor correction (PFC) has attracted significant research interest in recent years. To reduce input current harmonics in power supplies, PFC shapes the AC line current to be sinusoidal in phase with the line voltage. With this approach, electronic equipment produces less harmonics to pollute the power system and, hence, decreases the necessity of power generation. Passive PFCs are commonly used in low-cost applications, because they only require a couple of simple components. However, the corrected input power factors of passive PFCs are not higher than 0.95 . For high-quality requirements, products need to produce less harmonics and their input power factors are usually higher than 0.97. In these cases, active PFCs are introduced by specialist power electronics with control circuitry [1]. These controllers sense the line current and calculate the pulse duty for the power switch to shape the line current so that it becomes almost purely sinusoidal. The power factors of these techniques are extremely high and often up to 0.99 [2]. The harmonics of the line current are much less than when using passive PFCs.

During the past decade, several active single-phase power-factor correction topologies have been presented in the literature [1, 2]. Among them, boost PFC is the most popular circuit because of low cost and high efficiency. The input inductor current of the PFCs can be controlled in continuous or discontinuous conduction mode [3]. Discontinuous conduction mode (DCM) is usually used only in small power supplies for good quality high-frequency current ripples [4]. Continuous conduction mode (CCM) is widely utilised in high quality, medium and high power applications [5-7]. A large enough input inductor may limit the current harmonics around the switching frequency yet, in the meanwhile, this large inductor may arouse significant low-frequency distortion $[2,8]$. For this reason, the rela-

\section{(C) IEE, 2001}

IEE Proceedings online no. 20010120

DOI: 10.1049/ip-epa:20010120

Paper first received 20th January and in revised form 31st May 2000

The authors are with the Power Electronics Laboratory, Department of Electrical Engineering, National Taiwan University, Taipei, Taiwan 10617 tionship between the input inductance and the low-frequency current distortion of the CCMPFC is quantitatively analysed. An upper bound of the boost inductance is imposed on the input inductance to comply with the current harmonics regulations.

In this paper, the input voltage and current of PFCs are normalised into a per-unit system. Under the standard perunit system, the input line current of the PFC is analysed with its harmonic components in detail. Design examples of the CCMPFC demonstrate the upper limits of boost inductance. Finally, experimental results verify the theoretical derivations.

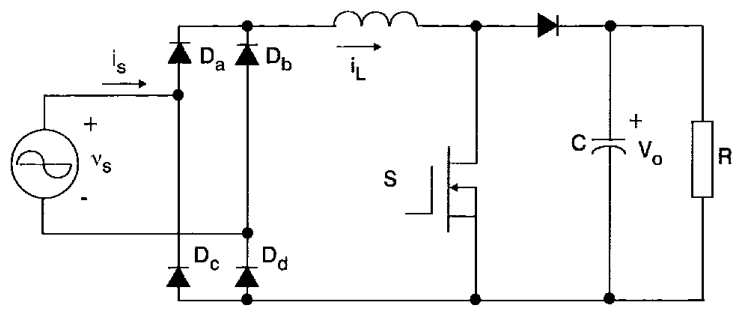

Fig. 1 Single-phase boost power-factor corrector

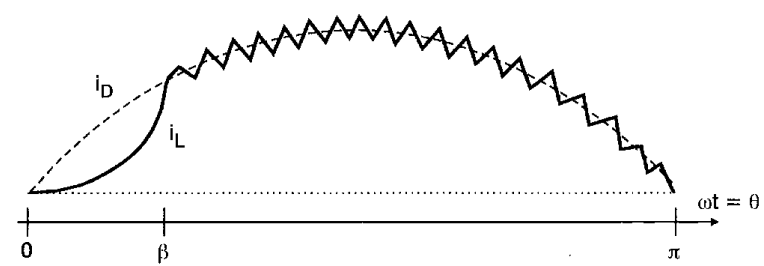

Fig.2 Inductor-current waveform of the CCMPFC

\section{Analysis of line current distortion}

A single-phase boost PFC is depicted in Fig. 1. The inductor current waveform which operates in CCM is demonstrated in Fig. 2. The switch $\mathrm{S}$ in Fig. 1 is considered an ideal switch, and the bulk capacitor $\mathrm{C}$ is large enough to maintain the output voltage of the $\mathrm{PFC}$ as a constant. There are two major components of current distortions in this waveform compared with an ideal sinusoidal current waveform $i_{D}$. The first one is the low-frequency component. During the starting of each half-cycle $(0<\omega t<\beta)$,

IEE Proc-Electi. Power Appl., Vol. 148, No. 2, March 2001 
the instantaneous value of the input voltage, $v_{S}$, is too small to force the inductor current to follow the sinusoidal reference. Until the time instant $\beta$, the inductor current gains enough variation rate, $\mathrm{d} i / \mathrm{d} t$, to rejoin the demand current $i_{D}$. The second component is the high-frequency switching current ripples. In low-frequency analysis, it is assumed that the high-frequency ripples are easily absorbed by the line filters, therefore it can be neglected.

For convenience, the input voltage and line current can be normalised as a per-unit system as follows:

$$
V_{b a s e}=V_{S}, \quad I_{\text {base }}=\frac{V_{S}}{\omega L}, \quad Z_{\text {base }}=\omega L, \quad \omega t=\theta
$$

Per-unit circuit parameters are located with a ${ }^{i \sim}$ above the symbol.

The circuit parameters used in this paper are:

$a_{n} \quad=n$th sine coefficient of Fourier series

$b_{n} \quad=n$th cosine coefficient of Fourier series

$D \quad=$ switching duty cycle

$f_{S} \quad=$ switching frequency

$\tilde{I}_{D} \quad=$ RMS of per-unit demand line current

$\tilde{i}_{n(R M S)}=$ RMS of $n$th order harmonic line current

$\tilde{i}_{S}=$ per-unit instantaneous line current

$\tilde{i}_{S(R M S)}=$ RMS of per-unit instantaneous line current

$\mathrm{PF} \quad=$ power factor

$P_{s} \quad=$ average input power

$\tilde{P}_{S} \quad=$ average per-unit input power

$V_{O}=$ output voltage of $\mathrm{PFC}$

$V_{S} \quad=$ RMS input voltage

$\tilde{v}_{S}=$ per-unit instantaneous input voltage

$V_{S(\text { min })}=$ minimum RMS input voltage

$\tilde{v}_{S(R M S)}=$ RMS of per-unit instantaneous line voltage

$\beta=$ rejoined angle of line current and demand current

$\Delta I \quad=$ maximum peak-to-peak current ripple

$\theta=$ instantaneous angle of input voltage or line current

In Fig. 2, the per-unit ideal sinusoidal demand current is

$$
\tilde{i}_{D}=\sqrt{2} \tilde{I}_{D} \sin \theta \quad 0 \leq \theta \leq \pi
$$

The per-unit input voltage and current waveforms can be expressed as follows:

$$
\begin{array}{cc}
\tilde{v}_{S}=\sqrt{2} \sin \theta & 0 \leq \theta \leq \pi \\
\tilde{i}_{S}=\sqrt{2}(1-\cos \theta) & 0 \leq \theta \leq \beta \\
\tilde{i}_{S}=\sqrt{2} \tilde{I}_{D} \sin \theta & \beta \leq \theta \leq \pi
\end{array}
$$

At angle $\theta=\beta, \tilde{i}_{S}$ in eqns. 3 and 4 rejoin, $\tilde{I}_{D}$ is given by

$$
\tilde{I}_{D}=\tan \left(\frac{\beta}{2}\right)
$$

This per-unit input current can be expressed by its harmonics with Fourier series representation:

$$
\tilde{i}_{S}=\sqrt{2} \sum_{n=1}^{\infty}\left[a_{n} \sin (n \theta)+b_{n} \cos (n \theta)\right]
$$

The fundamental components of the input current are [2]:

$$
a_{1}=\frac{\tan \frac{\beta}{2}}{\pi}(\pi+\sin \beta-\beta)
$$

$$
b_{1}=\frac{1}{\pi}(\sin \beta-\beta)
$$

The harmonic components can also be expressed in terms of $\beta$ :

For even $n, a_{n(\text { even })}=0$ and $b_{n(\text { even })}=0$

For odd $n$, the harmonic components are

$$
\begin{aligned}
a_{n(\text { odd })}= & -\frac{2 \tan \frac{\beta}{2}}{\pi}\left\{\frac{\sin [(n-1) \beta]}{2(n-1)}-\frac{\sin [(n+1) \beta]}{2(n+1)}\right\} \\
& +\frac{2}{\pi}\left\{\frac{\cos [(n-1) \beta]-1}{2(n-1)}+\frac{\cos [(n+1) \beta]-1}{2(n+1)}\right\} \\
& -\frac{2}{n \pi}\{[\cos (n \beta)]-1\} \\
b_{n(\text { odd })}= & \frac{2 \tan \frac{\beta}{2}}{\pi} \\
& \times\left\{\frac{\cos [(n-1) \beta]-1}{2(n-1)}-\frac{\cos [(n+1) \beta]-1}{2(n+1)}\right\} \\
& -\frac{2}{\pi}\left\{\frac{\sin [(n-1) \beta]}{2(n-1)}+\frac{\sin [(n+1) \beta]}{2(n+1)}\right\} \\
& +\frac{2}{n \pi} \sin (n \beta)
\end{aligned}
$$

In eqns. 9 and 10, $n$ must be an odd number and is equal to or larger than 3 . The per-unit RMS of the $n$th order harmonic current is given by

$$
\tilde{i}_{n(R M S)}=\sqrt{a_{n}^{2}+b_{n}^{2}}
$$

The average per-unit input power is given by

$$
\tilde{P}_{s}=\frac{1}{\pi} \int_{0}^{\pi} \tilde{v}_{S} \tilde{i}_{S} d \theta=\frac{\tan \frac{\beta}{2}}{\pi}(\pi+\sin \beta-\beta)
$$

A linear approximation for per-unit input power and $\beta$ is

$$
\tilde{P}_{s} \approx 0.5 \beta
$$

The relation between the per-unit power and actual power is

$$
P_{s}=V_{b a s e} I_{b a s e} \tilde{p}_{s}=\frac{V_{s}^{2}}{\omega L} \tilde{p}_{s}
$$

The power factor can be calculated by

$$
P F=\frac{\tilde{P}_{S}}{\tilde{v}_{S(R M S)^{2}} \tilde{\tilde{i}}_{S(R M S)}}
$$

where

$$
\begin{gathered}
\tilde{i}_{S(R M S)} \\
=\sqrt{\frac{2}{\pi}\left[\frac{3 \beta}{2}-2 \sin \beta+\frac{\sin (2 \beta)}{4}+\left(\frac{\pi-\beta}{2}+\frac{\sin (2 \beta)}{4}\right) \tan ^{2} \frac{\beta}{2}\right]} \\
\tilde{v}_{S(R M S)}=1
\end{gathered}
$$

From the above analysis, the rejoined angle $\beta$ is an important design parameter to determine limits of input inductance for given harmonic constraints. Fig. 3 shows the curves of the $n$th harmonics and angle $\beta$.

To determine the minimum value of the inductance, the relation between the inductor current ripple and inductance was presented in [5]: 


$$
L_{\text {min }}=\frac{\sqrt{2} V_{S(\min )} D}{\Delta I f_{S}}
$$

where

$$
D=\frac{V_{0}-\sqrt{2} V_{S(\min )}}{V_{o}}
$$

Take a $3000 \mathrm{~W}$ CCMPFC, for example: assume the output voltage of the $\mathrm{PFC}$ is $400 \mathrm{~V}$; the minimum input voltage of the PFC is $85 \mathrm{VRMS}$; the switching frequency is $50 \mathrm{kHz}$ and the maximum switching current ripple is $5 \mathrm{~A}$. According to eqns. 18 and 19, the minimum value of the input inductance is $0.43 \mathrm{mH}$. If the $3 \mathrm{rd}$ harmonic current cannot exceed $0.3 \mathrm{~A}$, the maximum boost inductance is $0.96 \mathrm{mH}$. The inductance is valid from $0.43 \mathrm{mH}$ to $0.96 \mathrm{mH}$. Two practical design examples of the CCMPFC are given in the next Section.

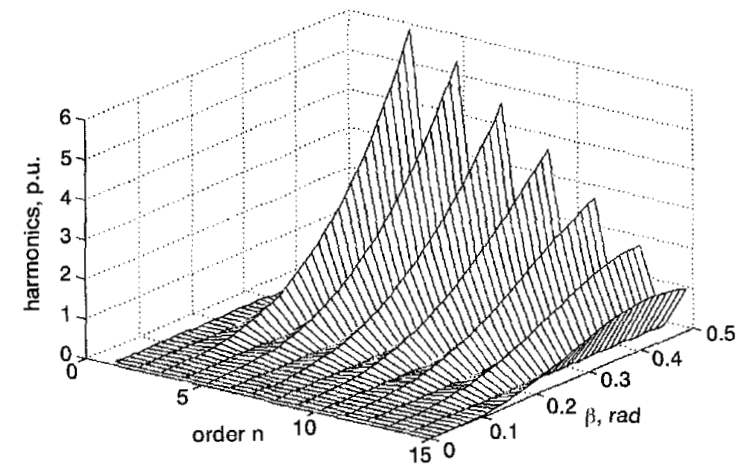

Fig.3 Curves of the nth harmonics and $\beta$

\section{Design examples}

A 1000W CCMPFC allows AC line voltage from $90 \sim 260 \mathrm{~V}$. Its output is $400 \mathrm{VDC}$. The limits of the input harmonic currents are:

power factor $>0.99$

3 rd harmonic $<2 \%$ of RMS current

5 th harmonic $<1 \%$ of RMS current

7 th harmonic $<0.5 \%$ of RMS current

9th harmonic $<0.35 \%$ of RMS current

According to Fig. 3, the harmonics are not linearly distributed with the angle $\beta$, so it requires a detail search to find the minimum inductance. A 3-D plot between the input power, input voltage and required $\beta$ is shown in Fig. 4 . The maximum boost inductances for CCMPFC from $90 \sim 260 \mathrm{~V}$ and $100 \sim 1000 \mathrm{~W}$ are listed in Table 1. The plot

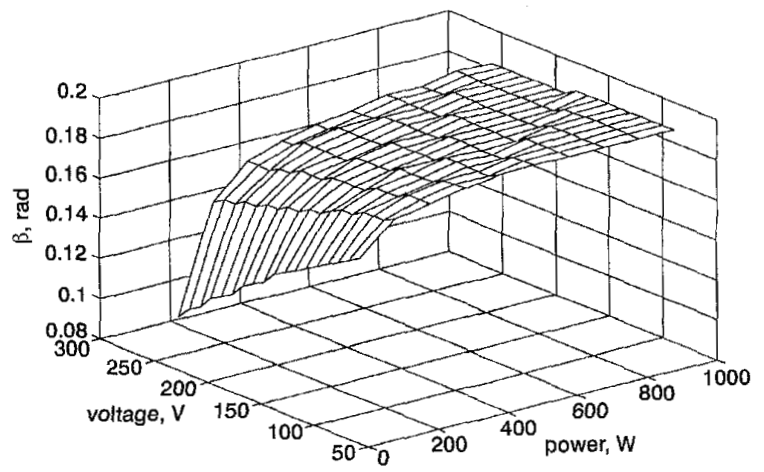

Fig. 4 Curves of the input voltage, input power and $\beta$ between the input power, input voltage and required inductances is shown in Fig. 5. From Table 1 and Fig. 5, the minimum input inductance $L$ is $2 \mathrm{mH}$ at $90 \mathrm{VAC}$ and $1000 \mathrm{~W}$. The switching frequency is selected at $50 \mathrm{kHz}$ to reduce the switching loss and the size of the boost inductor. From eqns. 15 and 16, the power factor of the PFC can be calculated. If the maximum switching current ripple is $1 \mathrm{~A}$, from eqns. 18 and 19, the lower limit of the inductance is $1.7 \mathrm{mH}$. The inductance is valid from $1.7 \mathrm{mH}$ to $2 \mathrm{mH}$ for low-frequency and high-frequency harmonic limits.

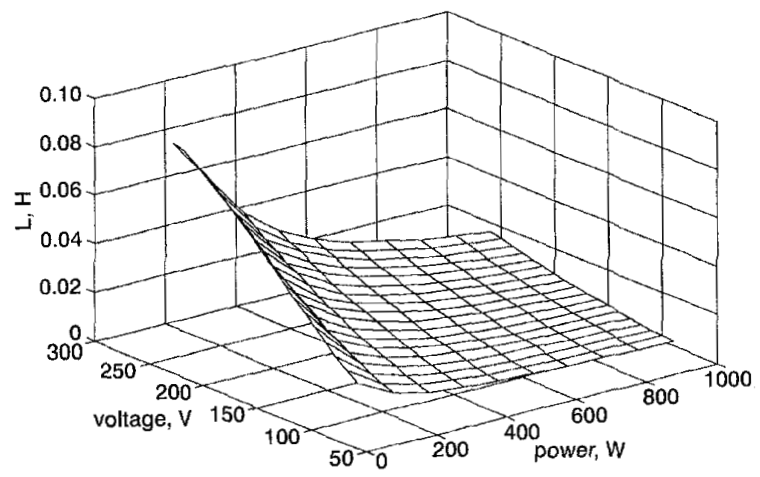

Fig.5 Curves of the input voltage, input power and $L$

To demonstrate the distortion of the CCMPFC, another $500 \mathrm{~W}$ CCMPFC allowing AC line voltage from $90 \sim 260 \mathrm{~V}$ is also established. Its output is $400 \mathrm{VDC}$. The limits of the input harmonic currents are:

power factor $>0.99$

3rd harmonic $<5 \%$ of RMS current

5 th harmonic $<3 \%$ of RMS current

7 th harmonic $<2 \%$ of RMS current

9 th harmonic $<1 \%$ of RMS current

The maximum boost inductance $L$ of the $500 \mathrm{~W}$ CCMPFC is $7.5 \mathrm{mH}$ at $90 \mathrm{VAC}$ and $500 \mathrm{~W}$. The switching frequency is also $50 \mathrm{kHz}$. The inductance is valid from $1.7 \mathrm{mH}$ to $7.5 \mathrm{mH}$.

\section{Experimental results}

Two single-phase CCMPFCs are implemented for the current harmonics and distortion tests. The boost inductance of $1000 \mathrm{~W}$ CCMPFC is selected as $2 \mathrm{mH}$. Measured RMS input harmonics and power factors are listed in Table 2. The boost inductance of 500W CCMPFC is selected at

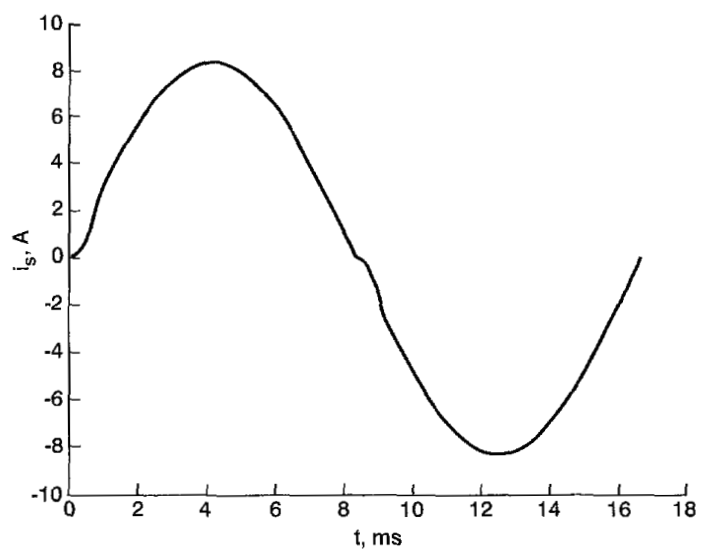

Fig. 6 Simulated input-current waveform at 90VAC/500 W 
Table 1: Calculated boost inductances $L(\mathrm{MH})$ at rated currents $(\mathrm{A})$

\begin{tabular}{|c|c|c|c|c|c|c|c|c|c|c|}
\hline & $100 \mathrm{~W}$ & $200 W$ & $300 \mathrm{~W}$ & $400 W$ & $500 \mathrm{~W}$ & $600 \mathrm{~W}$ & $700 \mathrm{~W}$ & $800 W$ & $900 \mathrm{~W}$ & $1000 \mathrm{~W}$ \\
\hline $90 \mathrm{~V}$ & $\begin{array}{l}17.31 \\
1.11 \mathrm{~A}\end{array}$ & $\begin{array}{l}9.51 \\
2.22 \mathrm{~A}\end{array}$ & $\begin{array}{l}6.45 \\
3.33 \mathrm{~A}\end{array}$ & $\begin{array}{l}4.92 \\
4.44 \mathrm{~A}\end{array}$ & $\begin{array}{l}4.01 \\
5.56 \mathrm{~A}\end{array}$ & $\begin{array}{l}3.34 \\
6.67 \mathrm{~A}\end{array}$ & $\begin{array}{l}2.86 \\
7.78 \mathrm{~A}\end{array}$ & $\begin{array}{l}2.51 \\
8.89 \mathrm{~A}\end{array}$ & $\begin{array}{l}2.23 \\
10.00 \mathrm{~A}\end{array}$ & $\begin{array}{l}2.01 \\
11.11 \mathrm{~A}\end{array}$ \\
\hline $100 \mathrm{~V}$ & $\begin{array}{l}20.95 \\
1.00 \mathrm{~A}\end{array}$ & $\begin{array}{l}11.53 \\
2.00 \mathrm{~A}\end{array}$ & $\begin{array}{l}7.96 \\
3.00 \mathrm{~A}\end{array}$ & $\begin{array}{l}6.08 \\
4.00 \mathrm{~A}\end{array}$ & $\begin{array}{l}4.86 \\
5.00 \mathrm{~A}\end{array}$ & $\begin{array}{l}4.12 \\
6.00 \mathrm{~A}\end{array}$ & $\begin{array}{l}3.53 \\
7.00 \mathrm{~A}\end{array}$ & $\begin{array}{l}3.09 \\
8.00 \mathrm{~A}\end{array}$ & $\begin{array}{l}2.75 \\
9.00 \mathrm{~A}\end{array}$ & $\begin{array}{l}2.48 \\
10.00 \mathrm{~A}\end{array}$ \\
\hline $110 \mathrm{~V}$ & $\begin{array}{l}24.85 \\
0.91 \mathrm{~A}\end{array}$ & $\begin{array}{l}13.95 \\
1.82 \mathrm{~A}\end{array}$ & $\begin{array}{l}9.64 \\
2.73 \mathrm{~A}\end{array}$ & $\begin{array}{l}7.35 \\
3.64 \mathrm{~A}\end{array}$ & $\begin{array}{l}5.89 \\
4.55 \mathrm{~A}\end{array}$ & $\begin{array}{l}4.99 \\
5.45 \mathrm{~A}\end{array}$ & $\begin{array}{l}4.28 \\
6.36 \mathrm{~A}\end{array}$ & $\begin{array}{l}3.74 \\
7.27 \mathrm{~A}\end{array}$ & $\begin{array}{l}3.33 \\
8.18 \mathrm{~A}\end{array}$ & $\begin{array}{l}2.99 \\
9.09 \mathrm{~A}\end{array}$ \\
\hline $120 \mathrm{~V}$ & $\begin{array}{l}28.96 \\
0.83 A\end{array}$ & $\begin{array}{l}16.60 \\
1.67 \mathrm{~A}\end{array}$ & $\begin{array}{l}11.27 \\
2.50 \mathrm{~A}\end{array}$ & $\begin{array}{l}8.60 \\
3.33 \mathrm{~A}\end{array}$ & $\begin{array}{l}7.00 \\
4.17 \mathrm{~A}\end{array}$ & $\begin{array}{l}5.84 \\
5.00 \mathrm{~A}\end{array}$ & $\begin{array}{l}5.09 \\
5.83 A\end{array}$ & $\begin{array}{l}4.45 \\
6.67 \mathrm{~A}\end{array}$ & $\begin{array}{l}3.96 \\
7.50 \mathrm{~A}\end{array}$ & $\begin{array}{l}3.56 \\
8.33 \mathrm{~A}\end{array}$ \\
\hline $130 \mathrm{~V}$ & $\begin{array}{l}33.28 \\
0.77 \mathrm{~A}\end{array}$ & $\begin{array}{l}19.12 \\
1.54 \mathrm{~A}\end{array}$ & $\begin{array}{l}13.22 \\
2.31 \mathrm{~A}\end{array}$ & $\begin{array}{l}10.10 \\
3.08 \mathrm{~A}\end{array}$ & $\begin{array}{l}8.22 \\
3.85 \mathrm{~A}\end{array}$ & $\begin{array}{l}6.85 \\
4.62 \mathrm{~A}\end{array}$ & $\begin{array}{l}5.97 \\
5.38 \mathrm{~A}\end{array}$ & $\begin{array}{l}5.23 \\
6.15 \mathrm{~A}\end{array}$ & $\begin{array}{l}4.65 \\
6.92 \mathrm{~A}\end{array}$ & $\begin{array}{l}4.18 \\
7.69 \mathrm{~A}\end{array}$ \\
\hline $140 \mathrm{~V}$ & $\begin{array}{l}37.78 \\
0.71 \mathrm{~A}\end{array}$ & $\begin{array}{l}22.18 \\
1.43 \mathrm{~A}\end{array}$ & $\begin{array}{l}15.33 \\
2.14 \mathrm{~A}\end{array}$ & $\begin{array}{l}11.71 \\
2.86 \mathrm{~A}\end{array}$ & $\begin{array}{l}9.53 \\
3.57 \mathrm{~A}\end{array}$ & $\begin{array}{l}7.94 \\
4.29 \mathrm{~A}\end{array}$ & $\begin{array}{l}6.81 \\
5.00 \mathrm{~A}\end{array}$ & $\begin{array}{l}6.06 \\
5.71 \mathrm{~A}\end{array}$ & $\begin{array}{l}5.39 \\
6.43 \mathrm{~A}\end{array}$ & $\begin{array}{l}4.85 \\
7.14 \mathrm{~A}\end{array}$ \\
\hline $150 \mathrm{~V}$ & $\begin{array}{l}42.43 \\
0.67 \mathrm{~A}\end{array}$ & $\begin{array}{l}24.99 \\
1.33 \mathrm{~A}\end{array}$ & $\begin{array}{l}17.29 \\
2.00 \mathrm{~A}\end{array}$ & $\begin{array}{l}13.44 \\
2.67 \mathrm{~A}\end{array}$ & $\begin{array}{l}10.75 \\
3.33 \mathrm{~A}\end{array}$ & $\begin{array}{l}9.12 \\
4.00 \mathrm{~A}\end{array}$ & $\begin{array}{l}7.82 \\
4.67 \mathrm{~A}\end{array}$ & $\begin{array}{l}6.84 \\
5.33 \mathrm{~A}\end{array}$ & $\begin{array}{l}6.18 \\
6.00 \mathrm{~A}\end{array}$ & $\begin{array}{l}5.57 \\
6.67 \mathrm{~A}\end{array}$ \\
\hline $160 \mathrm{~V}$ & $\begin{array}{l}47.20 \\
0.62 \mathrm{~A}\end{array}$ & $\begin{array}{l}27.89 \\
1.25 \mathrm{~A}\end{array}$ & $\begin{array}{l}19.67 \\
1.88 \mathrm{~A}\end{array}$ & $\begin{array}{l}15.02 \\
2.50 \mathrm{~A}\end{array}$ & $\begin{array}{l}12.23 \\
3.12 \mathrm{~A}\end{array}$ & $\begin{array}{l}10.37 \\
3.75 \mathrm{~A}\end{array}$ & $\begin{array}{l}8.89 \\
4.38 \mathrm{~A}\end{array}$ & $\begin{array}{l}7.78 \\
5.00 \mathrm{~A}\end{array}$ & $\begin{array}{l}7.03 \\
5.62 \mathrm{~A}\end{array}$ & $\begin{array}{l}6.33 \\
6.25 \mathrm{~A}\end{array}$ \\
\hline $170 \mathrm{~V}$ & $\begin{array}{l}52.08 \\
0.59 \mathrm{~A}\end{array}$ & $\begin{array}{l}31.49 \\
1.18 \mathrm{~A}\end{array}$ & $\begin{array}{l}22.21 \\
1.76 \mathrm{~A}\end{array}$ & $\begin{array}{l}16.96 \\
2.35 \mathrm{~A}\end{array}$ & $\begin{array}{l}13.81 \\
2.94 \mathrm{~A}\end{array}$ & $\begin{array}{l}11.71 \\
3.53 \mathrm{~A}\end{array}$ & $\begin{array}{l}10.04 \\
4.12 \mathrm{~A}\end{array}$ & $\begin{array}{l}8.79 \\
4.71 \mathrm{~A}\end{array}$ & $\begin{array}{l}7.81 \\
5.29 \mathrm{~A}\end{array}$ & $\begin{array}{l}7.15 \\
5.88 \mathrm{~A}\end{array}$ \\
\hline $180 \mathrm{~V}$ & $\begin{array}{l}55.67 \\
0.56 \mathrm{~A}\end{array}$ & $\begin{array}{l}34.62 \\
1.11 \mathrm{~A}\end{array}$ & $\begin{array}{l}24.89 \\
1.67 \mathrm{~A}\end{array}$ & $\begin{array}{l}19.01 \\
2.22 \mathrm{~A}\end{array}$ & $\begin{array}{l}15.48 \\
2.78 \mathrm{~A}\end{array}$ & $\begin{array}{l}12.90 \\
3.33 \mathrm{~A}\end{array}$ & $\begin{array}{l}11.25 \\
3.89 \mathrm{~A}\end{array}$ & $\begin{array}{l}9.85 \\
4.44 \mathrm{~A}\end{array}$ & $\begin{array}{l}8.76 \\
5.00 \mathrm{~A}\end{array}$ & $\begin{array}{l}8.01 \\
5.56 \mathrm{~A}\end{array}$ \\
\hline $190 \mathrm{~V}$ & $\begin{array}{l}60.52 \\
0.53 \mathrm{~A}\end{array}$ & $\begin{array}{l}38.58 \\
1.05 \mathrm{~A}\end{array}$ & $\begin{array}{l}27.23 \\
1.58 \mathrm{~A}\end{array}$ & $\begin{array}{l}21.18 \\
2.11 \mathrm{~A}\end{array}$ & $\begin{array}{l}17.25 \\
2.63 \mathrm{~A}\end{array}$ & $\begin{array}{l}14.38 \\
3.16 \mathrm{~A}\end{array}$ & $\begin{array}{l}12.54 \\
3.68 \mathrm{~A}\end{array}$ & $\begin{array}{l}10.97 \\
4.21 \mathrm{~A}\end{array}$ & $\begin{array}{l}9.76 \\
4.74 \mathrm{~A}\end{array}$ & $\begin{array}{l}8.78 \\
5.26 \mathrm{~A}\end{array}$ \\
\hline $200 \mathrm{~V}$ & $\begin{array}{l}65.39 \\
0.50 \mathrm{~A}\end{array}$ & $\begin{array}{l}41.91 \\
1.00 \mathrm{~A}\end{array}$ & $\begin{array}{l}30.17 \\
1.50 \mathrm{~A}\end{array}$ & $\begin{array}{l}23.05 \\
2.00 \mathrm{~A}\end{array}$ & $\begin{array}{l}18.78 \\
2.50 \mathrm{~A}\end{array}$ & $\begin{array}{l}15.93 \\
3.00 \mathrm{~A}\end{array}$ & $\begin{array}{l}13.89 \\
3.50 \mathrm{~A}\end{array}$ & $\begin{array}{l}12.16 \\
4.00 \mathrm{~A}\end{array}$ & $\begin{array}{l}10.81 \\
4.50 \mathrm{~A}\end{array}$ & $\begin{array}{l}9.73 \\
5.00 \mathrm{~A}\end{array}$ \\
\hline $210 \mathrm{~V}$ & $\begin{array}{l}68.40 \\
0.48 A\end{array}$ & $\begin{array}{l}46.20 \\
0.95 \mathrm{~A}\end{array}$ & $\begin{array}{l}33.27 \\
1.43 \mathrm{~A}\end{array}$ & $\begin{array}{l}25.42 \\
1.90 \mathrm{~A}\end{array}$ & $\begin{array}{l}20.70 \\
2.38 \mathrm{~A}\end{array}$ & $\begin{array}{l}17.56 \\
2.86 \mathrm{~A}\end{array}$ & $\begin{array}{l}15.06 \\
3.33 \mathrm{~A}\end{array}$ & $\begin{array}{l}13.40 \\
3.81 \mathrm{~A}\end{array}$ & $\begin{array}{l}11.92 \\
4.29 \mathrm{~A}\end{array}$ & $\begin{array}{l}10.73 \\
4.76 \mathrm{~A}\end{array}$ \\
\hline $220 \mathrm{~V}$ & $\begin{array}{l}73.05 \\
0.45 \mathrm{~A}\end{array}$ & $\begin{array}{l}49.69 \\
0.91 \mathrm{~A}\end{array}$ & $\begin{array}{l}35.83 \\
1.36 \mathrm{~A}\end{array}$ & $\begin{array}{l}27.89 \\
1.82 \mathrm{~A}\end{array}$ & $\begin{array}{l}22.72 \\
2.27 \mathrm{~A}\end{array}$ & $\begin{array}{l}19.27 \\
2.73 \mathrm{~A}\end{array}$ & $\begin{array}{l}16.52 \\
3.18 \mathrm{~A}\end{array}$ & $\begin{array}{l}14.71 \\
3.64 \mathrm{~A}\end{array}$ & $\begin{array}{l}13.08 \\
4.09 \mathrm{~A}\end{array}$ & $\begin{array}{l}11.77 \\
4.55 \mathrm{~A}\end{array}$ \\
\hline $230 \mathrm{~V}$ & $\begin{array}{l}77.63 \\
0.43 \mathrm{~A}\end{array}$ & $\begin{array}{l}54.31 \\
0.87 \mathrm{~A}\end{array}$ & $\begin{array}{l}39.17 \\
1.30 \mathrm{~A}\end{array}$ & $\begin{array}{l}30.48 \\
1.74 \mathrm{~A}\end{array}$ & $\begin{array}{l}24.83 \\
2.17 \mathrm{~A}\end{array}$ & $\begin{array}{l}21.06 \\
2.61 \mathrm{~A}\end{array}$ & $\begin{array}{l}18.06 \\
3.04 \mathrm{~A}\end{array}$ & $\begin{array}{l}16.08 \\
3.48 \mathrm{~A}\end{array}$ & $\begin{array}{l}14.29 \\
3.91 \mathrm{~A}\end{array}$ & $\begin{array}{l}12.86 \\
4.35 \mathrm{~A}\end{array}$ \\
\hline $240 \mathrm{~V}$ & $\begin{array}{l}79.71 \\
0.42 \mathrm{~A}\end{array}$ & $\begin{array}{l}57.93 \\
0.83 \mathrm{~A}\end{array}$ & $\begin{array}{l}41.84 \\
1.25 \mathrm{~A}\end{array}$ & $\begin{array}{l}33.19 \\
1.67 \mathrm{~A}\end{array}$ & $\begin{array}{l}27.04 \\
2.08 \mathrm{~A}\end{array}$ & $\begin{array}{l}22.54 \\
2.50 \mathrm{~A}\end{array}$ & $\begin{array}{l}19.66 \\
2.92 \mathrm{~A}\end{array}$ & $\begin{array}{l}17.21 \\
3.33 \mathrm{~A}\end{array}$ & $\begin{array}{l}15.56 \\
3.75 \mathrm{~A}\end{array}$ & $\begin{array}{l}14.01 \\
4.17 \mathrm{~A}\end{array}$ \\
\hline $250 \mathrm{~V}$ & $\begin{array}{l}83.88 \\
0.40 \mathrm{~A}\end{array}$ & $\begin{array}{l}62.86 \\
0.80 \mathrm{~A}\end{array}$ & $\begin{array}{l}45.40 \\
1.20 \mathrm{~A}\end{array}$ & $\begin{array}{l}35.36 \\
1.60 A\end{array}$ & $\begin{array}{l}28.82 \\
2.00 \mathrm{~A}\end{array}$ & $\begin{array}{l}24.45 \\
2.40 \mathrm{~A}\end{array}$ & $\begin{array}{l}21.33 \\
2.80 \mathrm{~A}\end{array}$ & $\begin{array}{l}18.67 \\
3.20 \mathrm{~A}\end{array}$ & $\begin{array}{l}16.88 \\
3.60 \mathrm{~A}\end{array}$ & $\begin{array}{l}15.20 \\
4.00 \mathrm{~A}\end{array}$ \\
\hline $260 \mathrm{~V}$ & $\begin{array}{l}85.08 \\
0.38 \mathrm{~A}\end{array}$ & $\begin{array}{l}66.57 \\
0.77 \mathrm{~A}\end{array}$ & $\begin{array}{l}49.10 \\
1.15 \mathrm{~A}\end{array}$ & $\begin{array}{l}38.25 \\
1.54 \mathrm{~A}\end{array}$ & $\begin{array}{l}31.17 \\
1.92 \mathrm{~A}\end{array}$ & $\begin{array}{l}26.45 \\
2.31 \mathrm{~A}\end{array}$ & $\begin{array}{l}23.07 \\
2.69 \mathrm{~A}\end{array}$ & $\begin{array}{l}20.19 \\
3.08 \mathrm{~A}\end{array}$ & $\begin{array}{l}18.26 \\
3.46 \mathrm{~A}\end{array}$ & $\begin{array}{l}16.44 \\
3.85 \mathrm{~A}\end{array}$ \\
\hline
\end{tabular}

Table 2: Measured harmonics and power factor for $1000 \mathrm{~W}$ CCMPFC

\begin{tabular}{lllll}
\hline & Limits & Measured & Limits & Measured \\
\hline$V_{\text {in }} \mathrm{V}$ & - & 87.8 & - & 266.7 \\
$F_{1} \mathrm{~Hz}$ & - & 60 & - & 60 \\
$\mathrm{lin}_{\mathrm{n},} \mathrm{A}$ & - & 12.2 & - & 4.3 \\
$P_{\text {in, }} \mathrm{W}$ & - & 1071 & - & 1147 \\
$\mathrm{PF}$ & 0.99 & 0.999 & 0.99 & 0.99 \\
$\mathrm{THD}(\%)$ & - & 3.6 & - & 7.4 \\
Harmonic 2, A & - & 0.024 & - & 0.009 \\
Harmonic 3, A & 0.244 & 0.240 & 0.086 & 0.069 \\
Harmonic 4, A & - & 0.017 & - & 0.006 \\
Harmonic 5, A & 0.122 & 0.049 & 0.043 & 0.039 \\
Harmonic 6, A & - & 0.004 & - & 0.001 \\
Harmonic 7, A & 0.061 & 0.030 & 0.022 & 0.016 \\
Harmonic 8, A & - & 0.001 & - & 0.001 \\
Harmonic 9, A & 0.043 & 0.030 & 0.015 & 0.010 \\
\hline
\end{tabular}

$7 \mathrm{mH}$ to illustrate the input current distortion. The computer simulated input current waveform of the CCMPFC at 90 VAC before the tests, is shown in Fig. 6. The measured input current waveform of the CCMPFC is shown in Fig. 7 to be the same as the simulated waveform. Measured RMS input harmonic currents and power factors are also listed in Table 3 . The harmonics all comply with the limits.

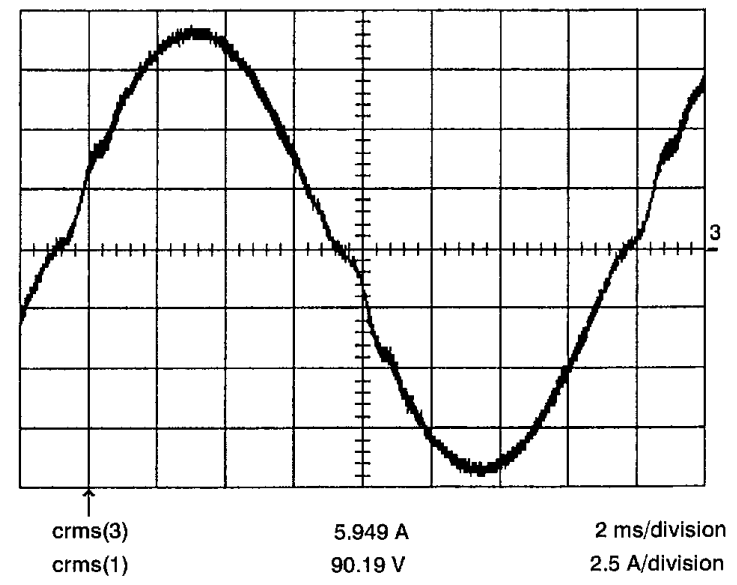

Fig.7 Measured input-current waveform at $90 \mathrm{VAC} / 5(0) \mathrm{W}$ 
Table 3: Measured harmonics and power factor for $500 \mathrm{~W}$ CCMPFC

\begin{tabular}{lllll}
\hline & Limits & Measured & Limits & Measured \\
\hline$V_{i n}, V$ & - & 90.7 & - & 265.6 \\
$F_{1} \mathrm{~Hz}$ & - & 60 & - & 60 \\
$l_{i n}, \mathrm{~A}$ & - & 5.95 & - & 2.2 \\
$P_{i n}, \mathrm{~W}$ & - & 534 & - & 584 \\
$\mathrm{PF}$ & 0.99 & 0.996 & 0.99 & 0.99 \\
THD (\%) & - & 4.3 & - & 9.6 \\
Harmonic 2, A & - & 0.005 & - & 0.009 \\
Harmonic 3, A & 0.298 & 0.087 & 0.11 & 0.12 \\
Harmonic 4, A & - & 0.005 & - & 0.002 \\
Harmonic 5, A & 0.179 & 0.074 & 0.066 & 0.036 \\
Harmonic 6, A & - & 0.002 & - & 0.003 \\
Harmonic 7, A & 0.119 & 0.064 & 0.044 & 0.033 \\
Harmonic 8, A & - & 0.001 & - & 0.001 \\
Harmonic 9, A & 0.059 & 0.052 & 0.011 & 0.005 \\
\hline
\end{tabular}

\section{Conclusions}

Single-phase CCMPFC for low-frequency input current distortion is presented. RMS input current harmonics are analysed in detail. Two CCMPFCs have been implemented for current harmonics and distortion tests. Experimental recordings have verified the theoretical derivations. The upper limits of input inductance of boost PFC are calculated under the constraints of harmonic currents. This is useful in high-quality power-supply design.

\section{References}

1 BOURGEOIS, J.M.: 'Circuits for power factor correction with regards to mains fittering'. SGS-Thomson application note, AN510, pp. $1-9$

2 SALMON, J.C.: 'Techniques for minimizing the input current distortion of current-controlled single-phase boost rectifiers', IEEE Trans. Power Electron., 1993, 8, (4), pp. 509-520

3 SEBASTIAN, J., JAUREGUIZAR, M., and UCEDA, J.: 'An overview of power factor correction in single-phase off-line power supply system'. Proceedings of the IEEE Industrial Electronics conference, 1994, pp. 1688-1693

4 ANDREYCAK, B.: 'Controlled on-time, zero current switched power factor correction technique'. Unitrode Switching Pregulated Power Supply Design Seminar Manual, 1993, pp I2-1-I2-11

5 DIXON, L.: 'High power factor switching preregulator design optimization'. Unitrode Switching Regulated Power Supply Design Seminar Manual, 1996, I3-1-I3-12

6 TODD, R C.: 'UC3854 controlled power factor correction circuit design'. Unitrode application note, U134, pp.3-269-3-289

7 COMANDATORE, G., and MORICONI, U.: 'Designing a high power factor switching preregulator with the L4981 continuous mode'. SGS-Thomson application note, AN628, pp.1-23

8 ZHOU, C., RIDLEY, R.B., and LEE, F.C.: 'Design and analysis of a hysteretic boost power factor correction circuit', IEEE Power Electron. Spec. Conf. Rec. 1990, pp. 800-807 